\title{
Optimal Designing of Variables Chain Sampling Plan by Minimizing the Average Sample Number
}

\author{
S. Balamurali and M. Usha \\ Department of Mathematics, Kalasalingam University, Krishnankoil, Tamil Nadu. 626 126, India \\ Correspondence should be addressed to S. Balamurali; sbmurali@rediffmail.com
}

Received 13 March 2013; Accepted 9 October 2013

Academic Editors: G.T.S. Ho, T. R. Kurfess, and I. Zeid

Copyright ( 2013 S. Balamurali and M. Usha. This is an open access article distributed under the Creative Commons Attribution License, which permits unrestricted use, distribution, and reproduction in any medium, provided the original work is properly cited.

\begin{abstract}
We investigate the optimal designing of chain sampling plan for the application of normally distributed quality characteristics. The chain sampling plan is one of the conditional sampling procedures and this plan under variables inspection will be useful when testing is costly and destructive. The advantages of this proposed variables plan over variables single sampling plan and variables double sampling plan are discussed. Tables are also constructed for the selection of optimal parameters of known and unknown standard deviation variables chain sampling plan for specified two points on the operating characteristic curve, namely, the acceptable quality level and the limiting quality level, along with the producer's and consumer's risks. The optimization problem is formulated as a nonlinear programming where the objective function to be minimized is the average sample number and the constraints are related to lot acceptance probabilities at acceptable quality level and limiting quality level under the operating characteristic curve.
\end{abstract}

\section{Introduction}

Although in recent years process control techniques and offline quality control methods have taken important roles, acceptance sampling procedures remain as a major tool for many practical quality control systems. In general, acceptance sampling is a statistical tool used to help make decisions concerning whether or not a batch or lot of products should be released for consumer use. That is, sampling plans are widely used in industries for estimating lot or process characteristics and for determining the disposition of lots. Variable sampling plans constitute one of the major areas of the theory and practice of acceptance sampling. The principle prerequisite for variables sampling is that the quality characteristic of interest is measured on a continuous scale. The primary advantage of the variables sampling plan is that the same operating characteristic (OC) curve can be obtained with a smaller sample size than would be required by an attributes sampling plan. Thus, a variables acceptance sampling plan would require less sampling. The variables sampling provides more information than the attributes sampling, and therefore the same protection is attained with partly considerable smaller sample size. When destructive testing is employed, variables sampling is particularly useful in reducing the costs of inspection.

However, it is to be pointed out that the variables sampling plans have some demerits compared to attributes sampling plans. The variables sampling plans are, of course, little bit more tedious to apply than the attributes sampling plans and the assumptions on which they are based may not be met or may not be known to be met. It is also obvious that the cost of inspecting each item by attributes is less than by variables. The lower cost may also be a result of the simpler procedure of recording only the number of units failing a test rather than recording variables measurements. However, in modern quality control systems, these reasons are no longer valid because measurements are made by automated test equipment. Even the simplest automated test equipment usually has the capability of the simple calculations and data accumulation needed for variables sampling. In one of his papers, Collani [1] criticized the variables sampling plans and argued that the acceptance sampling by variables is inappropriate if one is interested in the fraction nonconforming in incoming batches. But, at the same time, Seidel [2] has proved 
in one of his subsequent papers that the sampling by variables is always optimal.

The conventional variables sampling plans have been investigated by many authors and are available in the literature of acceptance sampling. Lieberman and Resnikoff [3] developed tables for the selection of plan parameters for various acceptable quality levels (AQL) for MIL-STD 414 scheme. Owen [4] developed variables sampling plans based on normal distribution when the standard deviation of the process is unknown. Bender Jr [5] considered variables sampling plans for assuring the product quality when the quality characteristic of the product follows normal distribution with unknown standard deviation and provided a procedure for calculating the noncentral $t$-distribution. Hamaker [6] has given a procedure for finding the parameters of the unknown sigma variables sampling plans from the known sigma variables sampling plans. Govindaraju and Soundararajan [7] developed tables for selecting the parameters of variables single sampling plans that match with the OC curves of MIL-STD 105D schemes. Bravo and Wetherill [8] developed a method for designing double sampling plans (DSP) by variables with OC curves matching with the OC curves of the equivalent single sampling plans. Schilling [9] has written an exclusive book on acceptance sampling which also deals with the conventional variables sampling plans. Baillie [10] developed tables for variables double sampling plans when the process standard deviation is unknown. Govindaraju and Balamurali [11] extended the chain sampling for variables inspection. However, they have not provided any tables for easy implementation of the plan. Also, they have restricted their discussion only with known standard deviation. Hence, this paper attempts to develop tables for easy application of chain sampling to variables inspection for a normally distributed quality characteristic for both known and unknown standard deviation cases.

\section{Chain Sampling Plan for Variables Inspection}

The concept of chain sampling was first introduced by Dodge [12] for the application of attribute quality characteristics. The characteristics and the properties of the chain sampling plan were investigated by many authors (see, for example, Soundararajan [13]). The chain sampling plan belongs to the group of conditional sampling procedures like multiple dependent (deferred) sampling plans (see Vaerst [14] and Wortham and Baker [15]). In these procedures, acceptance or rejection of a lot is based not only on the sample from that lot, but also on sample results from past lots. The chain sampling plan is applicable in the case of Type $B$ situations (i.e., sampling from a continuous process) where lots expected to be of the same quality are submitted for inspection serially in the order of production. The operating procedure and characteristics of the attributes chain sampling plan can be seen in Dodge [12].

The performance of an acceptance sampling plan can be measured by its OC curve which quantifies the risks of producer and consumers. It would be of interest to examine whether or not the shape of the variables plan is concave for good quality levels. If the variables plan possesses an unsatisfactory OC curve similar to that of the zero acceptance number attributes plan, then it is desirable to improve the shape of the curve. A detailed discussion on the shape of the OC curve of a single sampling variables plan with known standard deviation can be seen in Govindaraju and Kuralmani [16]. It is always necessary to increase the discriminating power of the OC curve. This can be achieved by increasing the sample size, but this may not be always possible particularly in costly and destructive testing. An alternative solution is to develop a conditional sampling procedure. Based on this idea, Govindaraju and Balamurali [11] extended the concept of chain sampling to variables inspection. As pointed out earlier, they have not provided any tables for the selection of parameters and also they have dealt only with known standard deviation case. This paper attempts to provide tables for the easy industrial application of this plan for both known and unknown standard deviation cases. It is also to be pointed out that Govindaraju and Balamurali [11] have given an approximate solution for finding the plans of unknown standard deviation case. But in this paper, we provide a different procedure for finding the unknown sigma chain sampling plan. The major advantage of this plan is to achieve better protection to the producer with minimum average sample number (ASN) and these aspects will be discussed in a separate section. The following assumptions should be valid for the application of the variables chain sampling plan.

(i) Lots are submitted for inspection serially in the order of production from a process that turns out a constant proportion nonconforming items.

(ii) The consumer has confidence in the supplier and there should be no reason to believe that a particular lot is poorer than the preceding lots.

In addition, the usual conditions for the application of single sampling variables plans with known or unknown standard deviation should also be valid. The operating procedures of the variables chain sampling plan are given in the next section.

\section{Operating Procedures of Variables Chain Sampling Plan}

3.1. Known Sigma Case. Suppose that the quality characteristic of interest has the upper specification limit $U$ and follows a normal distribution with unknown mean $\mu$ and known standard deviation $\sigma$. Then, the following two procedures of the variables chain sampling plan are proposed.

Step 1. From each submitted lot, take a random sample of size $n_{\sigma}$, say $\left(X_{1}, X_{2} \cdots X_{n_{\sigma}}\right)$, and compute $V=(U-\bar{X}) / \sigma$, where $\bar{X}=\left(1 / n_{\sigma}\right) \sum_{i=1}^{n_{\sigma}} X_{i}$.

Step 2. Accept the lot if $V \geq k_{\sigma}$ and reject the lot if $V<k_{\sigma}^{\prime}$. If $k_{\sigma}^{\prime} \leq V<k_{\sigma}$, then accept the current lot provided that the preceding $i_{\sigma}$ lots were accepted on the condition that $V \geq k_{\sigma}$ but reject, otherwise (note: $k_{\sigma}>k_{\sigma}^{\prime}$ ). 
In the case of lower specification limit $L$, the operating procedure is as follows.

Step 1. From each lot, take a random sample of size $n_{\sigma}$, say $\left(X_{1}, X_{2} \cdots X_{n_{\sigma}}\right)$, and compute $V=(\bar{X}-L) / \sigma$, where $\bar{X}=\left(1 / n_{\sigma}\right) \sum_{i=1}^{n_{\sigma}} X_{i}$.

Step 2. Accept the lot if $V \geq k_{\sigma}$ and reject the lot if $V<k_{\sigma}^{\prime}$. If $k_{\sigma}^{\prime} \leq V<k_{\sigma}$, then accept the current lot provided that the preceding $i_{\sigma}$ lots were accepted on the condition that $V \geq k_{\sigma}$ but reject, otherwise.

Thus, the proposed variables chain sampling plan is characterized by four parameters, namely, $n_{\sigma}, i_{\sigma}, k_{\sigma}$, and $k_{\sigma}^{\prime}$. If $k_{\sigma}=$ $k_{\sigma}^{\prime}$, then the proposed plan will reduce to the variables SSP. Also, when $i_{\sigma}$ tends to infinity, the proposed plan becomes variables SSP with parameters $n_{\sigma}$ and $k_{\sigma}$. It is to be pointed out that the chain sampling plan can be applied for inspection of lots which are submitted serially in the order of production or in the order of being submitted. The decision of current lot depends on the results of preceding lots. So, when $i_{\sigma} \geq$ 2 , we need to keep the records of results of previous lots. Of course, maintaining records of preceding lots may be a drawback of the chain sampling plan over the single sampling plan; however, this can be compensated by minimizing the inspection efforts in terms of minimum ASN with desired protection.

3.2. Unknown Sigma Case. Whenever the standard deviation is unknown, we may use the sample standard deviation $S$ instead of $\sigma$. In this case, the plan operates as follows.

Step 1. From each submitted lot, take a random sample of size $n_{S}$, say $\left(X_{1}, X_{2} \cdots X_{n_{S}}\right)$, and compute $V=$ $(U-\bar{X}) / S$, where $\bar{X}=\left(1 / n_{S}\right) \sum_{i=1}^{n_{S}} X_{i}$ and $S=$ $\sqrt{\sum\left(X_{i}-\bar{X}\right)^{2} /\left(n_{S}-1\right)}$.

Step 2. Accept the lot if $V \geq k_{S}$ and reject the lot if $V<k_{S}^{\prime}$. If $k_{S}^{\prime} \leq V<k_{S}$, then accept the current lot provided that the preceding $i_{S}$ lots were accepted on the condition that $V \geq k_{S}$.

Thus, the proposed unknown sigma variables chain sampling plan is characterized by four parameters, namely, $n_{S}, i_{S}, k_{S}$, and $k_{S}^{\prime}$. If $k_{S}=k_{S}^{\prime}$, then the proposed plan will reduce to the variables single sampling plan with unknown standard deviation.

\section{Designing Methodology of Variables Chain Sampling Plan}

Generally, variables sampling plans are designed based on two points on the OC curve, namely, $\left(p_{1}, 1-\alpha\right)$ and $\left(p_{2}, \beta\right)$, where $p_{1}$ is called the acceptable quality level (AQL), $p_{2}$ is the limiting quality level (LQL), $\alpha$ is the producer's risk, and $\beta$ is the consumer's risk. Any well-designed sampling plan must provide at least $(1-\alpha) \%$ probability of acceptance of a lot when the process fraction nonconforming is at AQL level and the sampling plan must also provide not more than $\beta \%$ probability of acceptance if the process fraction nonconforming is at the LQL level. Thus, the acceptance sampling plan must have its OC curve passing through two designated points (AQL, $1-\alpha$ ) and (LQL, $\beta$ ). Some other strategies are also followed to design the sampling plans besides the statistical based paradigm, which include Bayesian approach and economic based approach. For further details, readers may refer Chen and Lam [17], Ferrell and Chhoker [18], Chen [19], Chen et al. [20], Balamurali and Subramani [21], Vijayaraghavan and Sakthivel [22], Balamurali et al. [23], and Fallahnezhad and Aslam [24]. In this paper, we have followed the designing methodology based on two points on the OC curve approach. The variables chain sampling plan is designed based on the two points on the OC curve in the following manner.

The fraction nonconforming in a lot is given as

$$
p=1-\Phi\left(\frac{U-\mu}{\sigma}\right)
$$

where $\Phi(Y)$ is the cumulative distribution function of standard normal distribution and is given by

$$
\Phi(Y)=\int_{-\infty}^{Y} \frac{1}{\sqrt{2 \pi}} \exp \left(\frac{-Z^{2}}{2}\right) d Z .
$$

Here, the quality characteristic of interest is normally distributed with mean $\mu$ and standard deviation $\sigma$, and the unit is classified as nonconforming if it exceeds the upper specification limit $U$. So, the unknown mean $\mu$ can be determined if $p$ is specified. Let us define the standardized quality characteristic corresponding to the fraction conforming as

$$
Z_{p}=\Phi^{-1}(1-p)
$$

Then, the OC function of the variables chain sampling plan, which gives the proportion of lots that are expected to be accepted for given product quality, $p$, is given by (see Govindaraju and Balamurali [11])

$$
\begin{aligned}
P_{a}(p)= & \operatorname{Pr}(V \geq k) \\
& +\left[\operatorname{Pr}\left(V \geq k^{\prime}\right)-\operatorname{Pr}(V \geq k)\right][\operatorname{Pr}(V \geq k)]^{i},
\end{aligned}
$$

where $\operatorname{Pr}(V \geq k)$ is the probability of accepting a lot based on a single sample with parameters $(n, k)$ and $\operatorname{Pr}\left(V<k^{\prime}\right)$ is the probability of rejecting a lot based on a single sample with parameters $\left(n, k^{\prime}\right)$. Under Type $B$ situation (i.e., a series of lots of the same quality), forming lots of $N$ items from a process and then drawing random sample of size $n$ from these lots is equivalent to drawing random samples of size $n$ directly from the process. Hence, the derivation of the OC function is straightforward.

The probability of acceptance of the chain sampling plan can also be written as

$$
P_{a}(p)=\Phi\left(w_{2}\right)+\left[\Phi\left(w_{1}\right)-\Phi\left(w_{2}\right)\right]\left[\Phi\left(w_{2}\right)\right]^{i}
$$

where $w_{1}=\left(Z_{p}-k^{\prime}\right) \sqrt{n}$ and $w_{2}=\left(Z_{p}-k\right) \sqrt{n}$.

It is to be pointed out that the parameters of the known sigma variables chain sampling plan are denoted by 
$\left(n_{\sigma}, k_{\sigma}^{\prime}\right.$, and $\left.k_{\sigma}\right)$ and for unknown sigma plan they are denoted by $\left(n_{S}, k_{S}^{\prime}\right.$, and $\left.k_{S}\right)$. The determination of parameters $\left(n_{S}, k_{S}^{\prime}\right.$, and $k_{S}$ ) of unknown sigma variables chain sampling plan is explained as follows. It is known that, for large samples, $\bar{X} \pm k_{S} S$ is approximately normally distributed with mean $\mu \pm k_{S} E(S)$ and variance $\left(\sigma^{2} / n\right)+k_{S} \operatorname{Var}(S)$ (see Duncan [25], and Balamurali and Jun [26]). That is,

$$
\bar{X}+k_{S} S \sim N\left(\mu+k_{S} \sigma, \frac{\sigma^{2}}{n}+k_{S}^{2} \frac{\sigma^{2}}{2 n}\right) .
$$

Therefore, the probability of accepting a lot at each repetition is given by

$$
\begin{aligned}
P\left(V \geq k_{S}\right) & =P\left\{\bar{X} \leq U-k_{S} S \mid p\right\} \\
& =P\left\{\bar{X}+k_{S} S \leq U \mid p\right\} \\
& =\Phi\left(\frac{U-k_{S} \sigma-\mu}{\left(\sigma / \sqrt{n_{S}}\right) \sqrt{1+\left(k_{S}^{2} / 2\right)}}\right) \\
& =\Phi\left(\left(Z_{p}-k_{S}\right) \sqrt{\frac{n_{S}}{1+\left(k_{S}^{2} / 2\right)}}\right) .
\end{aligned}
$$

If we let

$$
\begin{aligned}
w_{2 S}= & \left(\left(Z_{p}-k_{S}\right) \sqrt{\frac{n_{S}}{1+\left(k_{S}^{2} / 2\right)}}\right), \\
& \text { then } \operatorname{Pr}\left(V \geq k_{S}\right)=\Phi\left(w_{2 S}\right) .
\end{aligned}
$$

Similarly, if we let $w_{1 S}=\left(\left(Z_{p}-k_{S}^{\prime}\right) \sqrt{n_{S} /\left(1+\left(k_{S}^{\prime 2} / 2\right)\right)}\right)$, then we have

$$
\operatorname{Pr}\left(V<k_{S}^{\prime}\right)=1-\Phi\left(w_{1 S}\right)
$$

Hence, the lot acceptance probability for sigma unknown case is given by

$$
P_{a}(p)=\Phi\left(w_{2 S}\right)+\left[\Phi\left(w_{1 S}\right)-\Phi\left(w_{2 S}\right)\right]\left[\Phi\left(w_{2 S}\right)\right]^{i_{S}},
$$

where $w_{1 S}=\left(Z_{p}-k_{S}^{\prime}\right) \sqrt{n_{S}}$ and $w_{2 S}=\left(Z_{p}-k_{S}\right) \sqrt{n_{S}}$.

If (AQL, $1-\alpha)$ and (LQL, $\beta)$ are prescribed, then the OC function can be written as

$$
\begin{gathered}
\Phi\left(w_{21 S}\right)+\left[\Phi\left(w_{11 S}\right)-\Phi\left(w_{21 S}\right)\right]\left[\Phi\left(w_{21 S}\right)\right]^{i_{S}}=1-\alpha, \\
\Phi\left(w_{22 S}\right)+\left[\Phi\left(w_{12 S}\right)-\Phi\left(w_{22 S}\right)\right]\left[\Phi\left(w_{22 S}\right)\right]^{i_{S}}=\beta
\end{gathered}
$$

Here, $w_{11 S}$ is the value of $w_{1 S}$ at $p=p_{1}, w_{21 S}$ is the value of $w_{2 S}$ at $p=p_{1}, w_{12 S}$ is the value of $w_{1 S}$ at $p=p_{2}$, and $w_{22 S}$ is the value of $w_{2 S}$ at $p=p_{2}$.
We obtain $w_{11 S}, w_{21 S}, w_{12 S}$, and $w_{22 S}$, respectively, by

$$
\begin{aligned}
& w_{11 S}=\left(\left(Z_{p 1}-k_{S}^{\prime}\right) \sqrt{\frac{n_{S}}{1+\left(k_{S}^{\prime 2} / 2\right)}}\right), \\
& w_{21 S}=\left(\left(Z_{p_{1}}-k_{S}\right) \sqrt{\frac{n_{S}}{1+\left(k_{S}^{2} / 2\right)}}\right), \\
& w_{12 S}=\left(\left(Z_{p_{2}}-k_{S}^{\prime}\right) \sqrt{\frac{n_{S}}{1+\left(k_{S}^{\prime 2} / 2\right)}}\right), \\
& w_{22 S}=\left(\left(Z_{p_{2}}-k_{S}\right) \sqrt{\frac{n_{S}}{1+\left(k_{S}^{2} / 2\right)}}\right),
\end{aligned}
$$

where $Z_{p_{1}}$ is the value of $Z_{p}$ at AQL and $Z_{p_{2}}$ is the value of $Z_{p}$ at LQL. For given AQL and LQL, the parametric values of the unknown sigma variables chain sampling plan, namely, $n_{S}, i_{S}, k_{S}$, and $k_{S}^{\prime}$, are determined by satisfying the required producer and consumer conditions. Alternatively, we can determine the above parameters of the variables chain sampling plan to minimize the ASN at AQL, which is analogous to minimizing the average sample number in the variables repetitive group sampling plans and multiple dependent state sampling plan (see Balamurali et al. [27] and Balamurali and Jun [26]). Some of the authors have investigated the designing of sampling plans by using some other optimization techniques which are available in the literature (see, for example, Feldmann and Krumbholz [28], Krumbholz and Rohr [29, 30], Krumbholz et al. [31], and Duarte and Sariava $[32,33]$. The ASN for the chain sampling plan is the sample size only. Therefore, the following optimization problem is considered to determine those parameters.

$$
\begin{array}{ll}
\text { Minimize } & \operatorname{ASN}\left(p_{1}\right)=n_{S} \\
& n_{S}, i_{S}, k_{S}, k_{S}^{\prime} \\
\text { Subject to } & P_{a}\left(p_{1}\right) \geq 1-\alpha \\
& P_{a}\left(p_{2}\right) \leq \beta \\
& n_{S} \geq 2, i_{S} \geq 1, k_{S}+\delta \geq k_{S}^{\prime}+\delta \geq 0, \\
& \delta \geq 0, n_{S} \in N, i_{S} \in N .
\end{array}
$$

In the case of known sigma variable chain sampling plan, the OC function under the specified AQL and LQL conditions can be written as

$$
\begin{gathered}
\Phi\left(w_{21 \sigma}\right)+\left[\Phi\left(w_{11 \sigma}\right)-\Phi\left(w_{21 \sigma}\right)\right]\left[\Phi\left(w_{21 \sigma}\right)\right]^{i_{\sigma}}=1-\alpha, \\
\Phi\left(w_{22 \sigma}\right)+\left[\Phi\left(w_{12 \sigma}\right)-\Phi\left(w_{22 \sigma}\right)\right]\left[\Phi\left(w_{22 \sigma}\right)\right]^{i_{\sigma}}=\beta .
\end{gathered}
$$

Here, $w_{11 \sigma}$ is the value of $w_{1 \sigma}$ at $p=p_{1}, w_{21 \sigma}$ is the value of $w_{2 \sigma}$ at $p=p_{1}, w_{12 \sigma}$ is the value of $w_{1 \sigma}$ at $p=p_{2}$, and $w_{22 \sigma}$ is the value of $w_{2 \sigma}$ at $p=p_{2}$. That is,

$$
\begin{array}{ll}
w_{11 \sigma}=\left(Z_{p_{1}}-k_{\sigma}^{\prime}\right) \sqrt{n_{\sigma}}, & w_{21 \sigma}=\left(Z_{p_{1}}-k_{\sigma}\right) \sqrt{n_{\sigma}}, \\
w_{12 \sigma}=\left(Z_{p_{2}}-k_{\sigma}^{\prime}\right) \sqrt{n_{\sigma}}, & w_{22 \sigma}=\left(Z_{p_{2}}-k_{\sigma}\right) \sqrt{n_{\sigma}},
\end{array}
$$


where $Z_{p_{1}}$ is the value of $Z_{p}$ at AQL and $Z_{p_{2}}$ is the value of $Z_{p}$ at LQL. The following optimization problem is considered to determine the optimal parameters of known sigma variables sampling plan such as $n_{\sigma}, i_{\sigma}, k_{\sigma}$, and $k_{\sigma}^{\prime}$.

$$
\begin{array}{ll}
\text { Minimize } & \operatorname{ASN}\left(p_{1}\right)=n_{\sigma} \\
& n_{\sigma}, i_{\sigma}, k_{\sigma}, k_{\sigma}^{\prime} \\
\text { Subject to } & P_{a}\left(p_{1}\right) \geq 1-\alpha \\
& P_{a}\left(p_{2}\right) \leq \beta \\
& n_{\sigma} \geq 2, i_{\sigma} \geq 1, k_{\sigma}+\delta \geq k_{\sigma}^{\prime}+\delta \geq 0, \\
& \delta \geq 0, n_{\sigma} \in N, i_{\sigma} \in N .
\end{array}
$$

We may determine the parameters of the unknown sigma chain sampling plan and the known sigma chain sampling plan by solving the nonlinear equation given in (13) and (16), respectively. There may exist multiple solutions since there are four unknowns with only two equations. Generally, a sampling would be desirable if the required number of samples is small. So, in this paper, we consider the ASN as the objective function to be minimized with the probability of acceptance along with the corresponding producer's and consumer's risks as constraints. To solve the above nonlinear optimization problems given in (13) and (16), the sequential quadratic programming (SQP) proposed by Nocedal and Wright [34] can be used. Here, it is to be pointed out that the optimization problems given in (13) and (16) include continuous values of $k_{\sigma}^{\prime}$ (or $k_{S}^{\prime}$ ) and $k_{\sigma}$ (or $k_{S}$ ) and integer values of $n_{\sigma}$ (or $n_{S}$ ) and $i_{\sigma}\left(\right.$ or $\left.i_{S}\right)$. This kind of formalization leads to a problem of mixed integer programming (MINLP) type. The SQP method does not allow directly solving MINLP problems but only the nonlinear programming (NLP) structures. The strategy we have followed is to relax the discrete variables first and solve the NLP problem and then fix them to the upper integers and resolve the problem again now just to find $k_{\sigma}^{\prime}$ (or $k_{S}^{\prime}$ ) and $k_{\sigma}\left(\right.$ or $\left.k_{S}\right)$. In such a way, we have solved the NLP problem given above. The SQP is implemented in Matlab software using the routine "fmincon." By solving the NLP based on the procedure described above, the optimal parameters $\left(n_{\sigma}\right.$, $i_{\sigma}, k_{\sigma}$, and $\left.k_{\sigma}^{\prime}\right)$ for known sigma plan and the parameters $\left(n_{S}\right.$, $i_{S}, k_{S}$, and $k_{S}^{\prime}$ ) for unknown sigma plan are determined and these values are tabulated in Table 1 .

\section{Designing Examples}

5.1. Selection of Known Sigma Variables Chain Sampling Plan for Specified AQL and LQL. Table 1 is used to determine the parameters of the known $\sigma$ variables chain sampling plan for specified values of AQL and LQL when $\alpha=5 \%$ and $\beta=10 \%$. For example, if $p_{1}=2 \%, p_{2}=7 \%, \alpha=5 \%$, and $\beta=10 \%$, Table 1 gives the parameters as $n_{\sigma}=18, i_{\sigma}=3, k_{\sigma}^{\prime}=1.544$, and $k_{\sigma}=$ 1.779 .

For the above example, the plan is operated as follows.

From each submitted lot, take a random sample of size 18 and compute $V=(U-\bar{X}) / \sigma$, where $\bar{X}=(1 / 18) \sum_{i=1}^{18} X_{i}$.

Accept the lot if $V \geq 1.779$ and reject the lot if $V<1.544$. If $1.544 \leq V<1.779$, then accept the current lot provided that the preceding 3 lots were accepted on the condition that $V \geq 1.779$ with the sample size of 18 .

5.2. Selection of Unknown Sigma Variables Chain Sampling Plan for Specified AQL and LQL. As mentioned earlier, the unknown sigma variables chain sampling plan is operated as a known sigma variables chain sampling plan, but the parameters $n_{S}, i_{S}, k_{S}$, and $k_{S}^{\prime}$ are used in the place of $n_{\sigma}, i_{\sigma}, k_{\sigma}$, and $k_{\sigma}^{\prime}$, respectively. Table 1 can also be used for the selection of the parameters of the unknown $\sigma$ variables chain sampling plan for given values of AQL and LQL. Suppose that AQL = 0.0075 , LQL $=0.035 \%, \alpha=5 \%$, and $\beta=10 \%$. From Table 1 , the parameters of the variables chain sampling plan can be determined as $n_{S}=47, i_{S}=1, k_{S}^{\prime}=1.925$, and $k_{S}=2.190$.

5.3. Illustrative Example. To illustrate the implementation of the proposed sampling plan for the example given in Section 5.2, we consider case study data on STN-LCD manufacturing process given in $\mathrm{Wu}$ and Pearn [35]. The upper specification limit is given as $0.77 \mathrm{~mm}$. Here, we consider only 47 values randomly taken from the original data given in $\mathrm{Wu}$ and Pearn [35]. The data are shown below:

$\begin{array}{llllllllll}0.717 & 0.698 & 0.726 & 0.684 & 0.727 & 0.688 & 0.708 & 0.703 & 0.694 & 0.713 \\ 0.730 & 0.699 & 0.710 & 0.688 & 0.665 & 0.704 & 0.725 & 0.729 & 0.716 & 0.685 \\ 0.712 & 0.716 & 0.712 & 0.733 & 0.709 & 0.703 & 0.730 & 0.716 & 0.688 & 0.688 \\ 0.712 & 0.702 & 0.726 & 0.669 & 0.718 & 0.714 & 0.726 & 0.683 & 0.713 & 0.737 \\ 0.740 & 0.706 & 0.726 & 0.688 & 0.715 & 0.704 & 0.724 & & & \end{array}$

The implementation of the plan is shown below.

Step 1. Take a random sample of size 47. The data are given above.

Step 2. For this data, we calculate $\bar{X}=(1 / n) \sum_{i=1}^{n_{S}} X_{i}=$ 0.708915 and $S=\sqrt{\sum\left(X_{i}-\bar{X}\right)^{2} /(n-1)}=0.017583$.
Step 3. Calculate $V=(U-\bar{X}) / S=(0.77-$ $0.708915) / 0.017583=3.4741$.

Step 4. Since $V=3.4741>k_{S}=2.190$, we accept the current lot without considering the result of past lots.

Just for the sake of discussion, let us assume that the $V$ value (for different data sets) is calculated as 2.15 . In this case, 
TABLE 1: Variables chain sampling plans indexed by AQL and LQL for $\alpha=5 \%$ and $\beta=10 \%$.

\begin{tabular}{|c|c|c|c|c|c|c|c|c|c|}
\hline \multirow{2}{*}{$p_{1}$} & \multirow{2}{*}{$p_{2}$} & \multicolumn{4}{|c|}{ Known sigma } & \multicolumn{4}{|c|}{ Unknown sigma } \\
\hline & & $n_{\sigma}$ & $i_{\sigma}$ & $k_{\sigma}^{\prime}$ & $k_{\sigma}$ & $n_{S}$ & $i_{S}$ & $k_{S}^{\prime}$ & $k_{S}$ \\
\hline \multirow{15}{*}{0.001} & 0.0020 & 119 & 1 & 2.883 & 3.013 & 650 & 1 & 2.888 & 3.013 \\
\hline & 0.0025 & 68 & 2 & 2.810 & 2.965 & 361 & 1 & 2.834 & 2.984 \\
\hline & 0.003 & 46 & 1 & 2.776 & 2.961 & 242 & 1 & 2.774 & 2.964 \\
\hline & 0.004 & 31 & 2 & 2.740 & 2.885 & 147 & 1 & 2.716 & 2.921 \\
\hline & 0.005 & 21 & 2 & 2.610 & 2.860 & 103 & 1 & 2.637 & 2.897 \\
\hline & 0.006 & 19 & 4 & 2.597 & 2.807 & 84 & 2 & 2.599 & 2.829 \\
\hline & 0.007 & 15 & 3 & 2.534 & 2.789 & 72 & 3 & 2.532 & 2.792 \\
\hline & 0.008 & 13 & 3 & 2.495 & 2.765 & 62 & 3 & 2.518 & 2.768 \\
\hline & 0.009 & 12 & 3 & 2.521 & 2.736 & 51 & 2 & 2.470 & 2.765 \\
\hline & 0.010 & 12 & 6 & 2.517 & 2.697 & 45 & 2 & 2.425 & 2.750 \\
\hline & 0.012 & 9 & 4 & 2.331 & 2.686 & 40 & 3 & 2.414 & 2.694 \\
\hline & 0.015 & 7 & 3 & 2.237 & 2.657 & 34 & 4 & 2.387 & 2.637 \\
\hline & 0.020 & 7 & 6 & 2.414 & 2.539 & 27 & 5 & 2.335 & 2.565 \\
\hline & 0.025 & 6 & 6 & 2.370 & 2.485 & 20 & 3 & 2.197 & 2.552 \\
\hline & 0.030 & 5 & 6 & 2.264 & 2.454 & 19 & 5 & 2.231 & 2.476 \\
\hline \multirow{12}{*}{0.0025} & 0.004 & 230 & 2 & 2.658 & 2.738 & 1131 & 2 & 2.676 & 2.736 \\
\hline & 0.005 & 105 & 2 & 2.598 & 2.703 & 474 & 2 & 2.585 & 2.705 \\
\hline & 0.006 & 64 & 2 & 2.530 & 2.675 & 282 & 1 & 2.533 & 2.698 \\
\hline & 0.0075 & 39 & 2 & 2.441 & 2.641 & 171 & 1 & 2.464 & 2.669 \\
\hline & 0.010 & 26 & 3 & 2.379 & 2.579 & 106 & 1 & 2.430 & 2.610 \\
\hline & 0.012 & 19 & 2 & 2.335 & 2.555 & 76 & 1 & 2.321 & 2.601 \\
\hline & 0.015 & 16 & 4 & 2.296 & 2.491 & 55 & 1 & 2.237 & 2.572 \\
\hline & 0.020 & 12 & 4 & 2.260 & 2.425 & 38 & 1 & 2.139 & 2.529 \\
\hline & 0.025 & 10 & 6 & 2.206 & 2.366 & 32 & 2 & 2.163 & 2.413 \\
\hline & 0.030 & 8 & 5 & 2.114 & 2.334 & 29 & 5 & 2.107 & 2.342 \\
\hline & 0.035 & 7 & 6 & 2.307 & 2.297 & 25 & 5 & 2.092 & 2.302 \\
\hline & 0.040 & 6 & 6 & 1.884 & 2.274 & 21 & 5 & 1.952 & 2.282 \\
\hline \multirow{10}{*}{0.005} & 0.0075 & 259 & 1 & 2.434 & 2.524 & 1113 & 2 & 2.442 & 2.512 \\
\hline & 0.010 & 86 & 1 & 2.326 & 2.486 & 347 & 1 & 2.348 & 2.483 \\
\hline & 0.012 & 53 & 1 & 2.277 & 2.457 & 211 & 1 & 2.299 & 2.454 \\
\hline & 0.015 & 33 & 1 & 2.211 & 2.421 & 128 & 1 & 2.233 & 2.418 \\
\hline & 0.020 & 24 & 4 & 2.161 & 2.316 & 75 & 2 & 2.100 & 2.345 \\
\hline & 0.025 & 16 & 3 & 2.046 & 2.281 & 55 & 2 & 2.083 & 2.293 \\
\hline & 0.030 & 14 & 6 & 1.989 & 2.224 & 43 & 3 & 1.974 & 2.249 \\
\hline & 0.035 & 12 & 6 & 2.002 & 2.182 & 36 & 3 & 1.979 & 2.209 \\
\hline & 0.040 & 10 & 4 & 1.976 & 2.156 & 31 & 3 & 1.964 & 2.174 \\
\hline & 0.050 & 8 & 5 & 1.893 & 2.098 & 25 & 4 & 1.925 & 2.105 \\
\hline \multirow{10}{*}{0.0075} & 0.010 & 505 & 2 & 2.339 & 2.384 & 1817 & 1 & 2.329 & 2.394 \\
\hline & 0.012 & 173 & 1 & 2.259 & 2.369 & 690 & 2 & 2.278 & 2.353 \\
\hline & 0.015 & 78 & 1 & 2.185 & 2.335 & 286 & 1 & 2.182 & 2.337 \\
\hline & 0.020 & 43 & 2 & 2.146 & 2.251 & 133 & 1 & 2.083 & 2.293 \\
\hline & 0.025 & 29 & 5 & 2.008 & 2.198 & 83 & 1 & 2.003 & 2.258 \\
\hline & 0.030 & 19 & 2 & 1.968 & 2.178 & 61 & 1 & 1.984 & 2.214 \\
\hline & 0.035 & 17 & 5 & 1.888 & 2.123 & 47 & 1 & 1.925 & 2.190 \\
\hline & 0.040 & 13 & 3 & 1.837 & 2.107 & 41 & 3 & 1.851 & 2.111 \\
\hline & 0.050 & 10 & 3 & 1.792 & 2.052 & 28 & 2 & 1.756 & 2.081 \\
\hline & 0.060 & 9 & 5 & 1.792 & 1.982 & 26 & 4 & 1.815 & 1.990 \\
\hline
\end{tabular}


TABle 1: Continued.

\begin{tabular}{|c|c|c|c|c|c|c|c|c|c|}
\hline \multirow{2}{*}{$p_{1}$} & \multirow{2}{*}{$p_{2}$} & \multicolumn{4}{|c|}{ Known sigma } & \multicolumn{4}{|c|}{ Unknown sigma } \\
\hline & & $n_{\sigma}$ & $i_{\sigma}$ & $k_{\sigma}^{\prime}$ & $k_{\sigma}$ & $n_{S}$ & $i_{S}$ & $k_{S}^{\prime}$ & $k_{S}$ \\
\hline \multirow{11}{*}{0.010} & 0.015 & 248 & 1 & 2.212 & 2.257 & 786 & 1 & 2.187 & 2.267 \\
\hline & 0.020 & 74 & 1 & 2.090 & 2.220 & 247 & 1 & 2.071 & 2.226 \\
\hline & 0.025 & 42 & 1 & 2.027 & 2.177 & 135 & 1 & 2.011 & 2.186 \\
\hline & 0.030 & 27 & 1 & 1.906 & 2.161 & 93 & 2 & 1.954 & 2.124 \\
\hline & 0.035 & 21 & 2 & 1.846 & 2.096 & 66 & 1 & 1.900 & 2.125 \\
\hline & 0.040 & 18 & 3 & 1.804 & 2.054 & 53 & 2 & 1.846 & 2.066 \\
\hline & 0.045 & 15 & 3 & 1.762 & 2.027 & 46 & 3 & 1.811 & 2.026 \\
\hline & 0.050 & 13 & 3 & 1.731 & 2.001 & 39 & 2 & 1.824 & 2.004 \\
\hline & 0.060 & 11 & 5 & 1.662 & 2.942 & 30 & 3 & 1.730 & 1.955 \\
\hline & 0.070 & 9 & 5 & 1.573 & 1.903 & 26 & 4 & 1.728 & 1.898 \\
\hline & 0.080 & 7 & 3 & 1.486 & 1.891 & 21 & 5 & 1.579 & 1.869 \\
\hline \multirow{14}{*}{0.02} & 0.030 & 187 & 2 & 1.896 & 1.976 & 528 & 1 & 1.886 & 1.991 \\
\hline & 0.035 & 96 & 2 & 1.835 & 1.945 & 272 & 2 & 1.831 & 1.946 \\
\hline & 0.040 & 59 & 1 & 1.779 & 1.939 & 169 & 2 & 1.770 & 1.920 \\
\hline & 0.045 & 45 & 2 & 1.749 & 1.889 & 117 & 1 & 1.734 & 1.919 \\
\hline & 0.050 & 34 & 2 & 1.693 & 1.868 & 88 & 1 & 1.683 & 1.903 \\
\hline & 0.060 & 22 & 2 & 1.563 & 1.833 & 63 & 2 & 1.670 & 1.820 \\
\hline & 0.070 & 18 & 3 & 1.544 & 1.779 & 44 & 2 & 1.577 & 1.792 \\
\hline & 0.080 & 14 & 3 & 1.434 & 1.749 & 36 & 3 & 1.525 & 1.745 \\
\hline & 0.090 & 13 & 4 & 1.502 & 1.697 & 27 & 2 & 1.423 & 1.737 \\
\hline & 0.10 & 10 & 2 & 1.436 & 1.691 & 22 & 1 & 1.386 & 1.766 \\
\hline & 0.11 & 10 & 4 & 1.457 & 1.632 & 23 & 4 & 1.476 & 1.636 \\
\hline & 0.12 & 7 & 1 & 1.235 & 1.725 & 17 & 1 & 1.379 & 1.699 \\
\hline & 0.13 & 8 & 5 & 1.360 & 1.580 & 17 & 4 & 1.356 & 1.596 \\
\hline & 0.15 & 6 & 4 & 1.110 & 1.560 & 14 & 5 & 1.279 & 1.544 \\
\hline \multirow{13}{*}{0.030} & 0.040 & 341 & 2 & 1.771 & 1.821 & 1027 & 5 & 1.771 & 1.816 \\
\hline & 0.045 & 157 & 1 & 1.711 & 1.811 & 416 & 2 & 1.704 & 1.799 \\
\hline & 0.050 & 96 & 1 & 1.650 & 1.795 & 253 & 1 & 1.679 & 1.789 \\
\hline & 0.060 & 52 & 2 & 1.566 & 1.736 & 126 & 1 & 1.585 & 1.760 \\
\hline & 0.070 & 33 & 1 & 1.508 & 1.728 & 81 & 1 & 1.539 & 1.724 \\
\hline & 0.080 & 26 & 3 & 1.427 & 1.657 & 62 & 2 & 1.514 & 1.659 \\
\hline & 0.090 & 23 & 5 & 1.449 & 1.609 & 44 & 2 & 1.401 & 1.641 \\
\hline & 0.100 & 17 & 3 & 1.378 & 1.593 & 36 & 2 & 1.384 & 1.609 \\
\hline & 0.110 & 13 & 2 & 1.268 & 1.588 & 29 & 2 & 1.305 & 1.590 \\
\hline & 0.120 & 11 & 1 & 1.270 & 1.605 & 26 & 3 & 1.274 & 1.549 \\
\hline & 0.130 & 11 & 4 & 1.204 & 1.514 & 23 & 3 & 1.289 & 1.519 \\
\hline & 0.150 & 10 & 6 & 1.273 & 1.443 & 18 & 3 & 1.230 & 1.475 \\
\hline & 0.200 & 6 & 5 & 0.950 & 1.365 & 12 & 5 & 1.134 & 1.354 \\
\hline \multirow{12}{*}{0.04} & 0.060 & 145 & 2 & 1.568 & 1.663 & 339 & 2 & 1.564 & 1.664 \\
\hline & 0.070 & 73 & 1 & 1.513 & 1.643 & 166 & 1 & 1.507 & 1.647 \\
\hline & 0.080 & 50 & 2 & 1.478 & 1.588 & 102 & 1 & 1.425 & 1.625 \\
\hline & 0.090 & 34 & 2 & 1.394 & 1.564 & 71 & 1 & 1.374 & 1.600 \\
\hline & 0.100 & 27 & 3 & 1.324 & 1.529 & 53 & 1 & 1.315 & 1.580 \\
\hline & 0.110 & 24 & 5 & 1.309 & 1.489 & 42 & 1 & 1.294 & 1.554 \\
\hline & 0.120 & 18 & 3 & 1.233 & 1.478 & 34 & 1 & 1.234 & 1.539 \\
\hline & 0.130 & 14 & 2 & 1.130 & 1.475 & 31 & 2 & 1.271 & 1.461 \\
\hline & 0.140 & 14 & 4 & 1.158 & 1.423 & 27 & 3 & 1.193 & 1.433 \\
\hline & 0.150 & 11 & 2 & 1.108 & 1.428 & 23 & 3 & 1.130 & 1.415 \\
\hline & 0.170 & 10 & 4 & 1.070 & 1.360 & 17 & 2 & 1.061 & 1.396 \\
\hline & 0.200 & 8 & 5 & 0.990 & 1.295 & 15 & 4 & 1.120 & 1.290 \\
\hline
\end{tabular}


TABle 1: Continued.

\begin{tabular}{|c|c|c|c|c|c|c|c|c|c|}
\hline \multirow{2}{*}{$p_{1}$} & \multirow{2}{*}{$p_{2}$} & \multicolumn{4}{|c|}{ Known sigma } & \multicolumn{4}{|c|}{ Unknown sigma } \\
\hline & & $n_{\sigma}$ & $i_{\sigma}$ & $k_{\sigma}^{\prime}$ & $k_{\sigma}$ & $n_{S}$ & $i_{S}$ & $k_{S}^{\prime}$ & $k_{S}$ \\
\hline \multirow{12}{*}{0.050} & 0.070 & 188 & 1 & 1.487 & 1.582 & 427 & 2 & 1.485 & 1.570 \\
\hline & 0.080 & 93 & 1 & 1.408 & 1.558 & 203 & 1 & 1.422 & 1.557 \\
\hline & 0.090 & 60 & 2 & 1.359 & 1.509 & 127 & 2 & 1.365 & 1.510 \\
\hline & 0.100 & 46 & 3 & 1.331 & 1.471 & 86 & 1 & 1.331 & 1.506 \\
\hline & 0.110 & 33 & 2 & 1.288 & 1.453 & 63 & 1 & 1.261 & 1.491 \\
\hline & 0.120 & 26 & 1 & 1.270 & 1.450 & 49 & 1 & 1.213 & 1.473 \\
\hline & 0.130 & 20 & 1 & 1.157 & 1.452 & 45 & 3 & 1.235 & 1.395 \\
\hline & 0.140 & 19 & 3 & 1.160 & 1.375 & 34 & 2 & 1.148 & 1.393 \\
\hline & 0.150 & 17 & 4 & 1.108 & 1.348 & 30 & 2 & 1.165 & 1.365 \\
\hline & 0.160 & 13 & 2 & 1.011 & 1.356 & 25 & 1 & 1.134 & 1.384 \\
\hline & 0.170 & 12 & 2 & 1.045 & 1.330 & 24 & 3 & 1.112 & 1.312 \\
\hline & 0.200 & 11 & 6 & 1.059 & 1.229 & 17 & 3 & 1.023 & 1.258 \\
\hline
\end{tabular}

TABLE 2: Variables chain sampling plans indexed by AQL and LQL for $\alpha=1 \%$ and $\beta=1 \%$.

\begin{tabular}{lcccccccc}
\hline$p_{1}$ & $p_{2}$ & $n_{\sigma}$ & $i_{\sigma}$ & $k_{\sigma}^{\prime}$ & $k_{\sigma}$ & $P_{a}\left(p_{1}\right)$ & $P_{a}\left(p_{2}\right)$ & ASN \\
\hline 0.001 & 0.005 & 79 & 1 & 2.82801 & 2.83801 & 0.99007 & 0.00991 \\
0.0025 & 0.01 & 91 & 1 & 2.56203 & 2.57203 & 0.99024 & 0.00956 \\
0.005 & 0.02 & 77 & 1 & 2.31004 & 2.32004 & 0.99012 & 0.00974 & 79 \\
0.0075 & 0.03 & 69 & 1 & 2.15205 & 2.16205 & 0.99003 & 0.00976 \\
0.01 & 0.04 & 64 & 1 & 2.03504 & 2.04504 & 0.99007 & 0.00929 \\
0.02 & 0.05 & 124 & 1 & 1.84403 & 1.85403 & 0.99018 & 0.00997 \\
0.03 & 0.06 & 193 & 1 & 1.71300 & 1.73200 & 0.99007 & 0.00977 \\
0.04 & 0.08 & 172 & 1 & 1.57299 & 1.58299 & 0.9906 & 0.00986 \\
0.05 & 0.10 & 156 & 1 & 1.45798 & 1.46798 & 0.99017 & 192 \\
\end{tabular}

we can accept the current lot provided previous lot must have been accepted with the condition that $V>k_{S}^{\prime}=1.925$. Otherwise, the current lot is rejected.

Further, it is to be pointed out that the proposed variables chain sampling plan is more efficient in terms of minimum ASN than the variables SSP for low values of producer's risk $(\alpha)$ and consumer's risk $(\beta)$. In order to prove this, we provide two tables. Table 2 gives the optimal parameters of variables chain sampling plan for some selected combinations of AQL and LQL and for $\alpha=1 \%$ and $\beta=1 \%$ and Table 3 gives the parameters of variables SSP under the same set of conditions. By comparing these two tables, one can easily observe that variable chain sampling plan involves minimum ASN compared to the variables SSP.

\section{Advantages of the Variables Chain Sampling Plan}

This section describes the advantages of the variables chain sampling plan over the conventional variables single sampling plan. Two acceptance sampling plans will be called equivalent when they possess nearly identical OC curves. A customary procedure for achieving such equivalency consists of constructing the sampling plans so that their OC curves coincide in two suitably chosen points, namely, $\left(p_{1}, 1-\alpha\right)$ and $\left(p_{2}, \beta\right)$. Suppose that, for given values of $p_{1}=0.5 \%, \alpha=$ $5 \%, p_{2}=1.5 \%$, and $\beta=10 \%$, one can find the parameters of the known sigma variables chain sampling plan from Table 1 as

(i) $n_{\sigma}=33, i_{\sigma}=1, k_{\sigma}^{\prime}=2.211$, and $k_{\sigma}=2.421 ; \mathrm{ASN}=33$.

For the same values of the AQL and LQL, we can determine the parameters of the single and double sampling variables plan (from Sommers [36]) as

(ii) $n_{\sigma}=53$ and $k_{\sigma}=2.35 ; \mathrm{ASN}=53$,

(iii) $n_{\sigma}=39, k_{a \sigma}=2.41$, and $k_{r \sigma}=2.31 ; \mathrm{ASN}=43$.

It can be observed that variables chain sampling plan achieves a reduction of over $38 \%$ in sample size than the variables single sampling plan and a reduction of $23 \%$ over double sampling plan with the same AQL and LQL conditions. This indicates that the variables chain sampling plan achieves the same OC curve with minimum sample size compared to the variables single and double sampling plans. Further, Figure 1 shows the OC curves of the variables chain sampling plans with parameters $n_{\sigma}=18, k_{\sigma}^{\prime}=1.544$, and $k_{\sigma}=1.779$ for different values of $i_{\sigma}$. This figure apparently shows that the variables chain sampling plan increases the probability of acceptance in the region of principal interest, that is, for good quality levels, and maintains the consumer's 
TABLE 3: Variables single sampling plans indexed by AQL and LQL for $\alpha=1 \%$ and $\beta=1 \%$.

\begin{tabular}{lcccccc}
\hline \multirow{2}{*}{$p_{1}$} & $p_{2}$ & \multicolumn{4}{c}{ Variables single sampling plan (known sigma) } \\
& & $n_{\sigma}$ & $k_{\sigma}$ & $P_{a}\left(p_{1}\right)$ & $P_{a}\left(p_{2}\right)$ & ASN \\
\hline 0.001 & 0.005 & 82 & 2.83305 & 0.99006 & 0.00992 & 0.00980 \\
0.0025 & 0.01 & 94 & 2.56703 & 0.99001 & 0.00970 & 94 \\
0.005 & 0.02 & 80 & 2.31505 & 0.99016 & 0.00932 & 72 \\
0.0075 & 0.03 & 72 & 2.15806 & 0.99004 & 0.00957 & 60 \\
0.01 & 0.04 & 66 & 2.03907 & 0.99015 & 0.00996 & 130 \\
0.02 & 0.05 & 130 & 1.84907 & 0.99018 & 0.00971 & 205 \\
0.03 & 0.06 & 205 & 1.71806 & 0.99009 & 0.00982 & 182 \\
0.04 & 0.08 & 182 & 1.57805 & 0.99007 & 0.00984 & 165 \\
0.05 & 0.10 & 165 & 1.46305 & 0.99025 & & \\
\hline
\end{tabular}

TABLE 4: ASN values of the variables SSP, DSP and variables chain sampling plans.

\begin{tabular}{|c|c|c|c|c|c|c|c|}
\hline \multirow{3}{*}{$p_{1}$} & \multirow{3}{*}{$p_{2}$} & \multicolumn{6}{|c|}{ Average sample number } \\
\hline & & \multicolumn{3}{|c|}{ Known sigma } & \multicolumn{3}{|c|}{ Unknown sigma } \\
\hline & & SSP & DSP & ChSP & SSP & DSP & ChSP \\
\hline 0.001 & 0.003 & 74 & 59.4 & 46 & 381 & 302.4 & 242 \\
\hline 0.0025 & 0.0075 & 62 & 50.1 & 39 & 267 & 214.2 & 171 \\
\hline 0.005 & 0.015 & 53 & 43.0 & 33 & 196 & 157.6 & 128 \\
\hline 0.0075 & 0.025 & 39 & 31.3 & 29 & 129 & 102.1 & 83 \\
\hline 0.01 & 0.05 & 19 & 14.9 & 13 & 54 & 41.5 & 39 \\
\hline 0.02 & 0.08 & 21 & 16.9 & 14 & 50 & 39.6 & 36 \\
\hline 0.03 & 0.09 & 30 & 24.0 & 23 & 66 & 52.5 & 44 \\
\hline 0.04 & 0.10 & 39 & 31.3 & 27 & 82 & 65.1 & 53 \\
\hline 0.05 & 0.12 & 39 & 32.0 & 26 & 76 & 60.9 & 49 \\
\hline
\end{tabular}

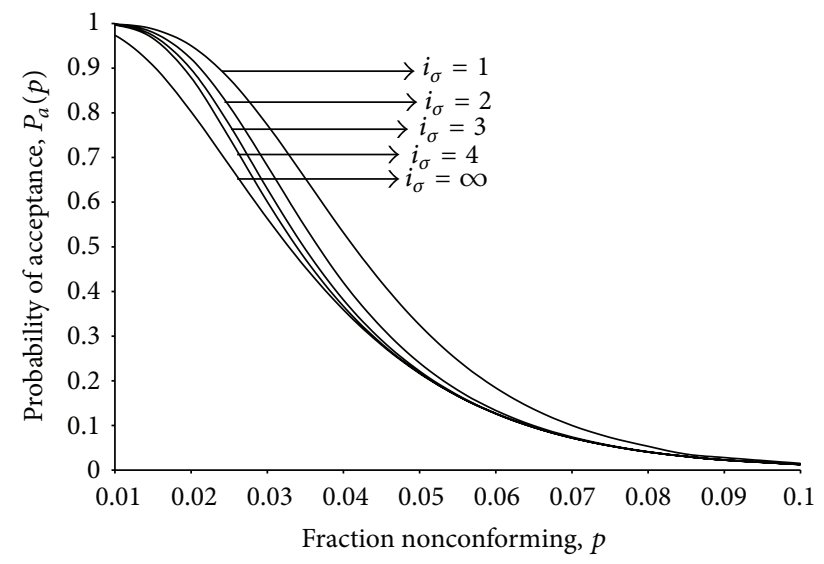

FIGURE 1: OC curves of a variables chain sampling plan for different $i_{\sigma}$ values.

risk at poor quality levels. This is also an important feature of the variables chain sampling plan.

Further, it is also to be noted that the variables chain sampling plan is economically superior to the double sampling plans in terms of average sample number (ASN). Obviously, a sampling plan having smaller ASN would be more desirable. The variables double or multiple sampling plans are not practically very useful. Variables sampling standards avoid presenting such plans due to increased complexity involved in operating them, but the variables chain sampling plan has no such complexity. Table 4 shows the ASNs for variables single sampling plan, variables double sampling plan along with variables chain sampling plan for some arbitrarily selected combinations of AQL and LQL. These ASN values are calculated at the producer's quality level for the known sigma plans. The ASN of the variables single and double sampling plans can be found in Sommers [36].

From this table, one can easily understand that the variables chain sampling plan will have minimum ASN when compared to the single and double sampling plans. Similar reduction in the ASN can be achieved for any combination of AQL and LQL values. This implies that variables chain sampling plan will give desired protection with minimum inspection so that the cost of inspection will greatly be reduced. Thus, the variables chain sampling plan provides better protection than the variables single and double sampling plans.

\section{Average Run Length of Variables Chain Sampling Plan}

Schilling [37] has pointed out that average run length (ARL) is a missing and meaningful measure for characterizing and evaluating the sampling plans under Type $B$ situations as in the process control procedures. The ARL gives an indication 
TABLE 5: ARLs of variables chain sampling plan for different $i_{\sigma}$ values.

\begin{tabular}{|c|c|c|c|c|c|}
\hline \multirow{2}{*}{$p$} & \multicolumn{5}{|c|}{ Average run length } \\
\hline & $i_{\sigma}=1$ & $i_{\sigma}=2$ & $i_{\sigma}=3$ & $i_{\sigma}=4$ & $i_{\sigma}=\infty$ \\
\hline 0 & $\infty$ & $\infty$ & $\infty$ & $\infty$ & $\infty$ \\
\hline 0.005 & 88768.34 & 71697.5 & 60133.4 & 51781.5 & 607.80 \\
\hline 0.01 & 868.88 & 554.66 & 410.18 & 327.19 & 38.29 \\
\hline 0.015 & 81.89 & 50.25 & 37.21 & 30.13 & 10.64 \\
\hline 0.02 & 20.07 & 12.66 & 9.77 & 8.25 & 5.06 \\
\hline 0.025 & 8.12 & 5.42 & 4.41 & 3.92 & 3.15 \\
\hline 0.03 & 4.41 & 3.14 & 2.70 & 2.50 & 2.29 \\
\hline 0.035 & 2.88 & 2.19 & 1.98 & 1.89 & 1.83 \\
\hline 0.04 & 2.14 & 1.73 & 1.62 & 1.58 & 1.56 \\
\hline 0.045 & 1.73 & 1.47 & 1.41 & 1.39 & 1.39 \\
\hline 0.05 & 1.48 & 1.32 & 1.28 & 1.28 & 1.28 \\
\hline 0.055 & 1.33 & 1.22 & 1.20 & 1.20 & 1.20 \\
\hline 0.06 & 1.23 & 1.15 & 1.15 & 1.14 & 1.14 \\
\hline 0.065 & 1.16 & 1.11 & 1.11 & 1.11 & 1.11 \\
\hline 0.07 & 1.11 & 1.08 & 1.08 & 1.08 & 1.08 \\
\hline 0.075 & 1.08 & 1.06 & 1.06 & 1.06 & 1.06 \\
\hline 0.08 & 1.06 & 1.04 & 1.04 & 1.04 & 1.04 \\
\hline 0.085 & 1.04 & 1.03 & 1.03 & 1.03 & 1.03 \\
\hline 0.09 & 1.03 & 1.02 & 1.02 & 1.02 & 1.02 \\
\hline 0.10 & 1.02 & 1.01 & 1.01 & 1.01 & 1.01 \\
\hline
\end{tabular}

of the expected number of samples until a decision is made. The ARL can be easily calculated once the probability of acceptance $\left(P_{a}(p)\right)$ of the plan is known for any process fraction nonconforming, $p$. It is clear that the distribution of the run length, $L$, follows the geometric distribution with probability mass function

$$
f_{G}(L)=\left[P_{a}(p)\right]^{L-1}\left[1-P_{a}(p)\right] .
$$

Its mean and variance are, respectively, given by

$$
\begin{gathered}
\mathrm{ARL}=E(L)=\frac{1}{\left(1-P_{a}(p)\right)} \\
\operatorname{Var}(L)=\frac{P_{a}(p)}{\left(1-P_{a}(p)\right)^{2}}
\end{gathered}
$$

where $P_{a}(p)$ is given in (5).

Table 5 gives the values of ARL of the chain sampling plan with $n_{\sigma}=16, k_{\sigma}^{\prime}=1.501$, and $k_{\sigma}=1.841$ for different values of $i_{\sigma}$. This table apparently shows that when the process fraction nonconforming is small, the ARL is high and for the increased values of fraction nonconforming, the ARL is low. By comparing the ARL values for different $i_{\sigma}$ values, when $i_{\sigma}$ increases, the ARL values reduce even for the lower fraction nonconforming. Also, it is clear from the table that $95 \%$ of the lots will be accepted at fraction nonconforming $2 \%$ by the variables chain sampling plan $\left(i_{\sigma}=1\right)$ at an average rate of 20 inspections, whereas with the single sampling plan $\left(i_{\sigma}=\infty\right)$, at $2 \%$ nonconforming, only $80 \%$ of lots will be accepted at an average rate of 5 inspections. Also, $90 \%$ of the lots will be rejected at the fraction nonconforming $7 \%$ by the variables chain sampling plan at an average rate of 1.11 inspections, and at the fraction nonconforming, $93 \%$ of the lots will be rejected by the single sampling plan at the rate of 1.08 inspections.

\section{Conclusions}

The purpose of this paper is to develop conditional sampling procedures for the inspection of normally distributed quality characteristics. Variables sampling plans generally require a smaller sample size than do attributes plans. If the OC curve of the variables plan is unsatisfactory, then its shape can be improved by chaining the past lot results. The proposed variables chain sampling plan is one of the variables conditional sampling plans which also ensure the protection against the consumer point of view. This plan is also simple to apply rather than double and multiple sampling variables plans. Also, this plan provides better protection than the conventional single and double sampling variables plans with minimum sample size. Such a variables chain sampling plan will be effective and useful for compliance testing. However, it is also to be pointed out that the variables chain sampling plan developed in this paper is based on the assumption that the quality characteristic of interest follows a normal distribution. Whenever the normality assumption is not true or invalid, using this variables chain sampling plan can be quite misleading. Hence, this study can be further extended for nonnormal distributions as future research. 


\section{Nomenclature}

$P: \quad$ Submitted lot quality; $q=1-p$

$p_{1}: \quad$ Acceptable quality level (AQL)

$p_{2}$ : $\quad$ Limiting quality level (LQL)

$\alpha: \quad$ Producer's risk

$\beta$ : $\quad$ Consumer's risk

$P_{a}(p)$ : The probability of lot acceptance when the fraction nonconforming is $p$

$n_{\sigma}: \quad$ Sample size for known sigma plan

$k_{\sigma}$ : Acceptance criterion for known sigma plan

$k_{\sigma}^{\prime}: \quad$ Rejection criterion for known sigma plan

$n_{S}: \quad$ Sample size for unknown sigma plan

$k_{s}$ : Acceptance criterion for unknown sigma plan

$k_{S}^{\prime}: \quad$ Rejection criterion for unknown sigma plan

$i_{\sigma}: \quad$ Number of preceding lots considered for accepting current lot for known sigma plan

$i_{S}: \quad$ Number of preceding lots considered for accepting current lot for unknown sigma plan

$V: \quad$ The value which is to be compared with acceptance criterion for making decision

$\bar{X}: \quad$ The sample mean

$S: \quad$ The sample standard deviation

$\sigma: \quad$ The population standard deviation

$\Phi(\cdot)$ : The cumulative distribution function of standard normal distribution

ASN: The average sample number

ARL: The average run length

SSP: Single sampling plan

DSP: Double sampling plan.

\section{Acknowledgment}

The authors would like to thank the anonymous reviewers and the editor for their valuable comments and suggestions which significantly improved the presentation of this paper.

\section{References}

[1] E. V. Collani, "A note on acceptance sampling for variables," Metrika, vol. 38, pp. 19-36, 1990.

[2] W. Seidel, "Is sampling by variables worse than sampling by attributes? A decision theoretic analysis and a new mixed strategy for inspecting individual lots," Sankhya B, vol. 59, pp. 96-107, 1997.

[3] G. J. Lieberman and G. J. Resnikoff, "Sampling plans for inspection by variables," Journal of the American Statistical Association, vol. 50, pp. 72-75, 1955.

[4] D. B. Owen, "Variables sampling plans based on the normal distribution," Technometrics, vol. 9, no. 3, pp. 417-423, 1967.

[5] A. Bender Jr., "Sampling by variables to control the fraction defective: part II," Journal of Quality Technology, vol. 7, no. 3, pp. 139-143, 1975.
[6] H. C. Hamaker, "Acceptance sampling for percent defective by variables and by attributes," Journal of Quality Technology, vol. 11, no. 3, pp. 139-148, 1979.

[7] K. Govindaraju and V. Soundararajan, "Selection of single sampling plans for variables matching the MIL-STD 105 scheme," Journal of Quality Technology, vol. 18, pp. 234-238, 1986.

[8] P. C. Bravo and G. B. Wetherill, “The matching of sampling plans and the design of double sampling plans," Journal of the Royal Statistical Society A, vol. 143, pp. 49-67, 1980.

[9] E. G. Schilling, Acceptance Sampling in Quality Control, Marcel Dekker, New York, NY, USA, 1982.

[10] D. H. Baillie, "Double sampling plans for inspection by variables when the process standard deviation is unknown," International Journal of Quality and Reliability Management, vol. 9, pp. 59-70, 1992.

[11] K. Govindaraju and S. Balamurali, "Chain sampling plan for variables inspection," Journal of Applied Statistics, vol. 25, no. 1, pp. 103-109, 1998.

[12] H. Dodge, "Chain sampling inspection plan," Industrial Quality Control, vol. 11, pp. 10-13, 1955.

[13] V. Soundararajan, "Procedures and tables for construction and selection of chain sampling plans (ChSP-1) - part I," Journal of Quality Technology, vol. 10, no. 2, pp. 56-60, 1978.

[14] R. Vaerst, "A procedure to construct multiple deferred state sampling plan," Methods of Operations Research, vol. 37, pp. 477$485,1982$.

[15] A. W. Wortham and R. C. Baker, "Multiple deferred state sampling inspection," International Journal of Production Research, vol. 14, no. 6, pp. 719-731, 1976.

[16] K. Govindaraju and V. Kuralmani, "A note on the operating characteristic curve of the known sigma single sampling variables plan," Communications in Statistics: Theory and Methods, vol. 21, pp. 2339-2347, 1992.

[17] J. Chen and Y. Lam, "Bayesian variable sampling plan for the Weibull distribution with type I censoring," Acta Mathematicae Applicatae Sinica, vol. 15, no. 3, pp. 269-280, 1999.

[18] W. G. Ferrell Jr. and A. Chhoker, "Design of economically optimal acceptance sampling plans with inspection error," Computers and Operations Research, vol. 29, no. 10, pp. 1283$1300,2002$.

[19] C. H. Chen, "Economic design of Dodge-Romig AOQL single sampling plans by variables with the quadratic loss function," Tamkang Journal of Science and Engineering, vol. 8, no. 4, pp. 313-318, 2005.

[20] J. Chen, K. Li, and Y. Lam, "Bayesian single and double variable sampling plans for the Weibull distribution with censoring," European Journal of Operational Research, vol. 177, no. 2, pp. 1062-1073, 2007.

[21] S. Balamurali and J. Subramani, "Economic design of SkSP-3 skip-lot sampling plans," International Journal of Mathematics and Statistics, vol. 6, no. 10, pp. 23-39, 2010.

[22] R. Vijayaraghavan and K. M. Sakthivel, "Chain sampling inspection plans based on Bayesian methodology," Economic Quality Control, vol. 26, no. 2, pp. 173-187, 2011.

[23] S. Balamurali, M. Aslam, C. H. Jun, and M. Ahmad, "Bayesian double sampling plan under gamma-poisson distribution," Research Journal of Applied Sciences, Engineering and Technology, vol. 4, no. 8, pp. 949-956, 2012.

[24] M. S. Fallahnezhad and M. Aslam, "A new economical design of acceptance sampling models using Bayesian inference," Accreditation and Quality Assurance, vol. 18, no. 3, pp. 187-195, 2013. 
[25] A. Duncan, Quality Control and Industrial Statistics, Richard D. Irwin, Homewood, Ill, USA, 5th edition, 1986.

[26] S. Balamurali and C. Jun, "Multiple dependent state sampling plans for lot acceptance based on measurement data," European Journal of Operational Research, vol. 180, no. 3, pp. 1221-1230, 2007.

[27] S. Balamurali, H. Park, C. H. Jun, K. J. Kim, and J. Lee, "Designing of variables repetitive group sampling plan involving minimum average sample number," Communications in Statistics: Simulation and Computation, vol. 34, no. 3, pp. 799809, 2005.

[28] B. Feldmann and W. Krumbholz, "ASN-minimax double sampling plans for variables," Statistical Papers, vol. 43, no. 3, pp. 361-377, 2002.

[29] W. Krumbholz and A. Rohr, "The operating characteristic of double sampling plans by variables when the standard deviation is unknown," Allgemeines Statistisches Archiv, vol. 90, no. 2, pp. 233-251, 2006.

[30] W. Krumbholz and A. Rohr, "Double ASN Minimax sampling plans by variables when the standard deviation is unknown," AStA Advances in Statistical Analysis, vol. 93, no. 3, pp. 281-294, 2009.

[31] W. Krumbholz, A. Rohr, and E. Vangjeli, "Minimax versions of the two-stage t test," Statistical Papers, vol. 53, no. 2, pp. 311-321, 2012.

[32] B. P. M. Duarte and P. M. Saraiva, "An optimization-based framework for designing acceptance sampling plans by variables for non-conforming proportions," International Journal of Quality and Reliability Management, vol. 27, no. 7, pp. 794-814, 2010.

[33] B. Duarte and P. Saraiva, "Optimal design of acceptance sampling plans by variables for non-conforming proportions when the standard deviation is unknown," Communications in Statistics: Simulation and Computation, vol. 42, pp. 1318-1342, 2013.

[34] J. Nocedal and S. J. Wright, Numerical Optimization, Springer, New York, NY, USA, 1999.

[35] C. Wu and W. L. Pearn, "A variables sampling plan based on Cpmk for product acceptance determination," European Journal of Operational Research, vol. 184, no. 2, pp. 549-560, 2008.

[36] D. J. Sommers, "Two-point double variables sampling plans," Journal of Quality Technology, vol. 13, pp. 25-30, 1981.

[37] E. G. Schilling, "Average run length and the OC curve of sampling plans," Quality Engineering, vol. 17, no. 3, pp. 399-404, 2005. 

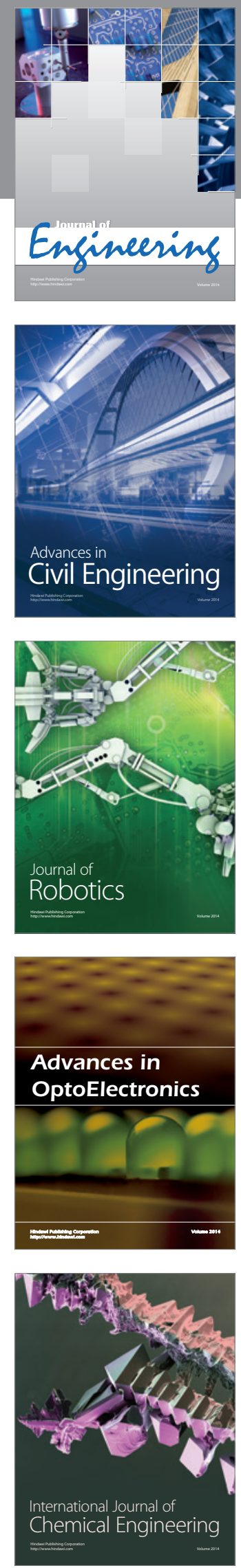

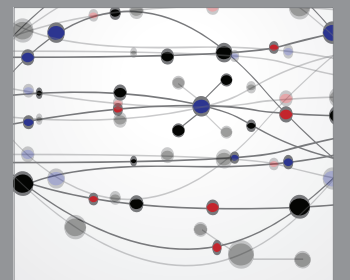

The Scientific World Journal
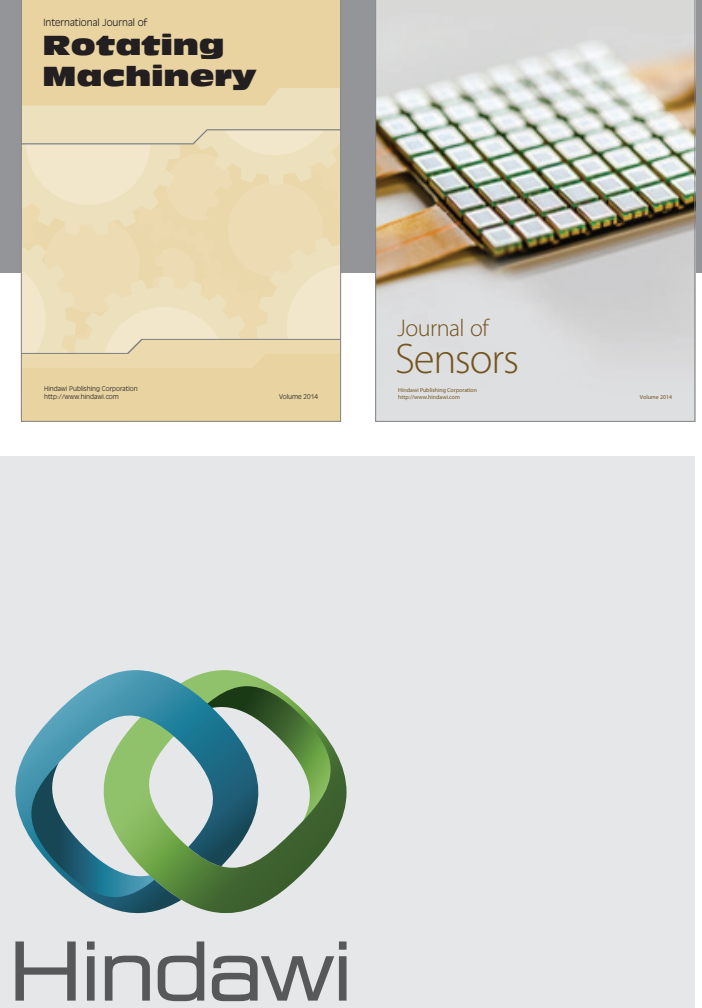

Submit your manuscripts at http://www.hindawi.com
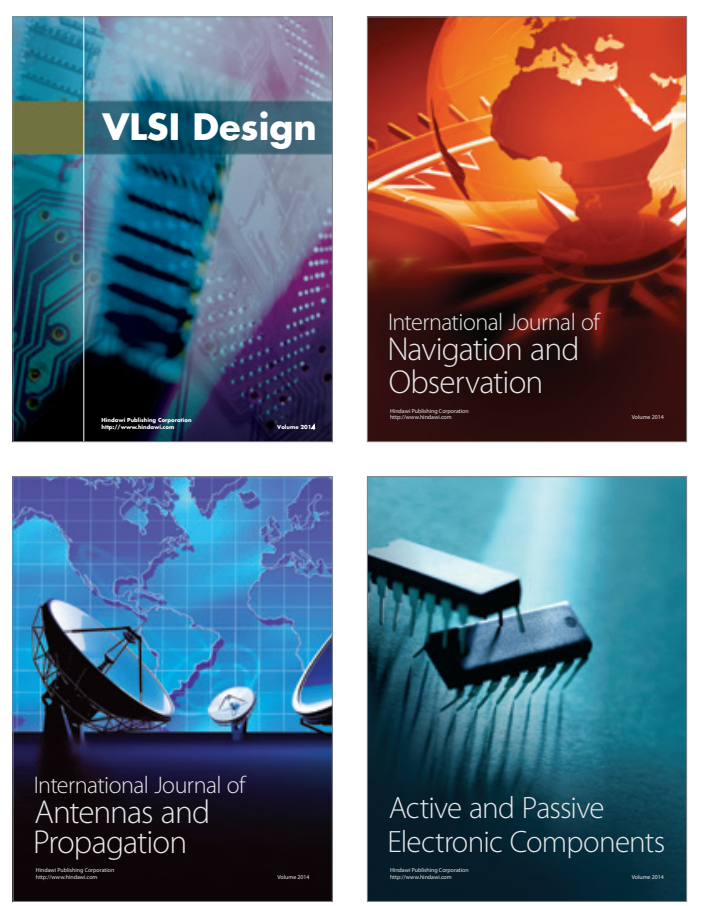
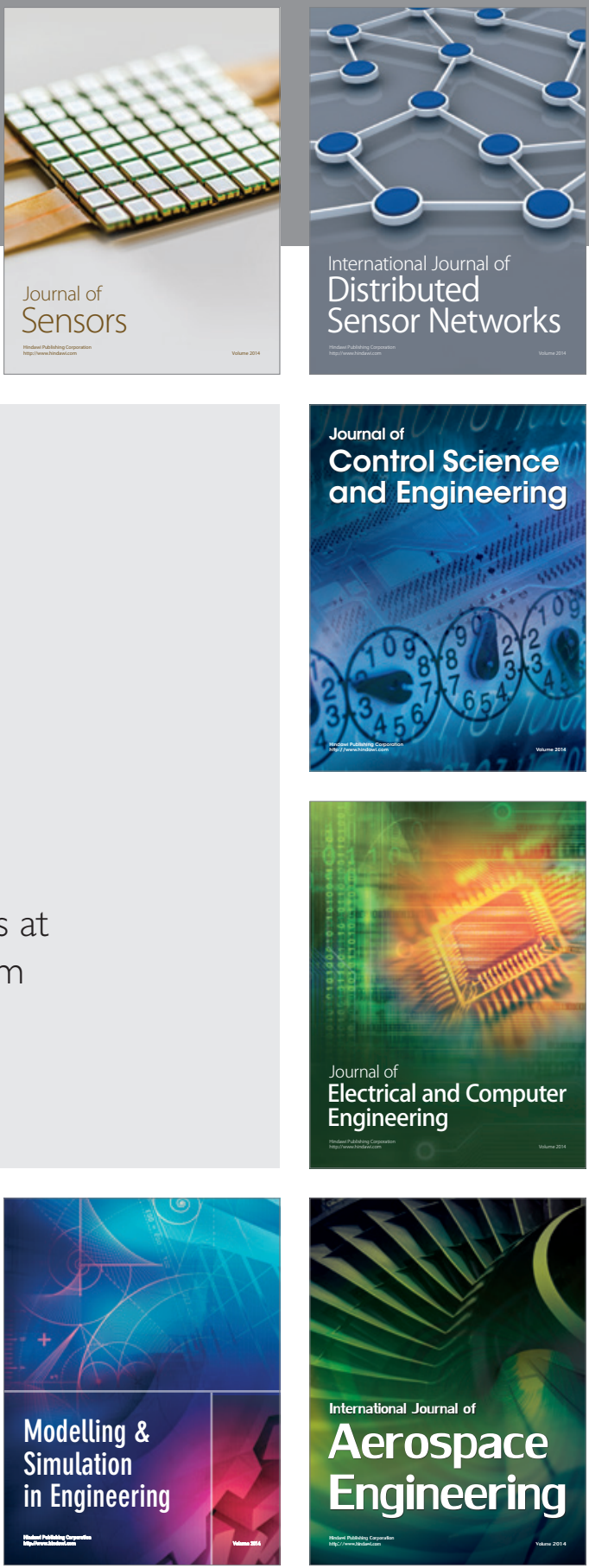

Journal of

Control Science

and Engineering
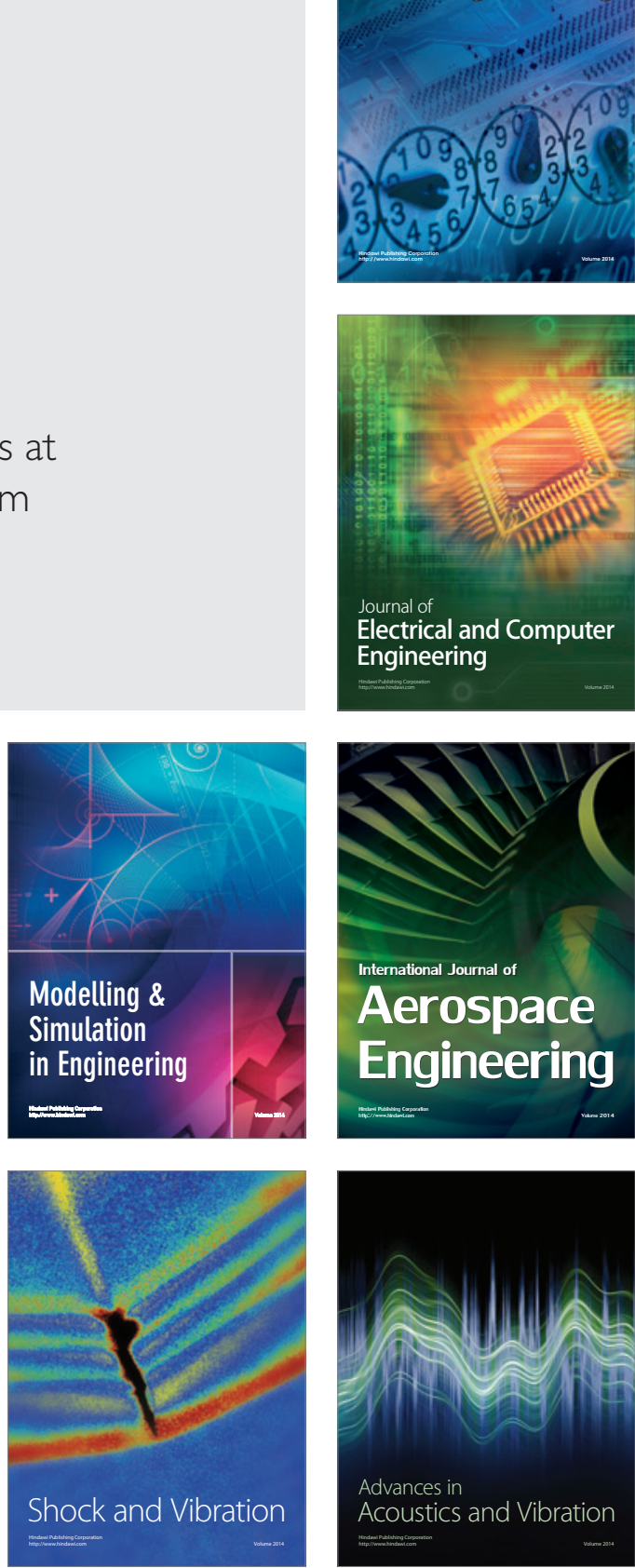